\title{
Considering Genre in Metal Music
}

(C) Benjamin Hillier, 2020. The definitive, peer reviewed, and edited version of this article is published in Metal Music Studies, Volume 6, Number 1, pp. 5-26, 2020, doi: https://doi.org/10.1386/mms_00002_1

Keywords: extreme metal, genre, heavy metal, metal musicology, metal studies, music analysis

\section{$\underline{\text { Introduction }}$}

In the late 1980s and early 1990s metal music experienced a widespread fracturing of musical styles resulting in the proliferation of a vast number of subgenres under the broad umbrella of 'metal'. This was especially prevalent amongst the collection of subgenres commonly referred to as 'extreme metal'. The differences between these subgenres are of deep importance to many fans and musicians for whom genre is an integral element of their musical identity and authenticity as 'metalheads' (Smialek, 2015). Despite this acknowledged importance, there is still a need to establish what Kahn-Harris calls 'a widely accepted vocabulary for identifying the constituent musical features of metal' (2011: 252). Metal studies broadly remains 'undertheorized and undermethdologized' (Weinstein, 2016: 29) with a need to clarify its academic rigour (Savigny and Schaap, 2018). It is these needs that this article seeks to address by analyzing the relevant literature discussing genre in metal and responding to the shortcomings identified.

This article proposes both a taxonomy for classifying metal subgenres and a model that provides a series of interconnected characteristics to assist in defining these subgenres. By clearly outlining ways in which we might organize the different 'levels' of subgenres, this article aims to provide a common vocabulary for metal studies scholars to use when analyzing genres and their relationships. The taxonomy discusses genre categories and the relationships between metal genres and outlines out the influences between metal genres as understood within metal communities and demonstrated by their characteristics. This takes the form of a 'genre web' to illustrate the relationships between metal genres as an alternative to the common 'family tree' types used elsewhere. The model consists of pitch, timbre, form, rhythm, and aesthetics. There are two main goals of this model. The first is to outline the specific ways in which these five layers might be used to define or characterize the various subgenres of metal. This is to assist theorists from any discipline in developing clear and specific definitions of metal subgenres that are based in their discrete musical characteristics and congruent with their present usages in metal communities. The second goal is to provide a framework through which analysis of metal music might be conducted. The model for understanding and classifying the attributes of metal subgenres seeks to develop an ontological method for defining subgenres by connecting the attributes that are considered definitive of a given metal subgenre. While this article does not provide specific definitions for any subgenres (as this level of analysis is beyond the scope of a single article), the model that it outlines aims to provide a means to achieve these definitions.

\section{Theorizing Genre in metal music}


Genre in metal is complex. Few other musical styles have such a variety of genres and subgenres within them and few place such importance on them (Spracklen, Brown, KahnHarris and Scott, 2016). This fragmentation of genres gives metal the appearance of a genre of music that is a mosaic of vaguely defined genres rather than a gestalt genre; indeed, viewing metal as a 'bricolage' has been a popular way of considering metal for decades (Weinstein, 2000[1991]; O'Boyle and Scott, 2016). The complexities of genre in metal are further compounded by the lack of musicological research in this area, as the subgenres of metal are rarely defined by clear, musical characteristics but instead by genealogies and fan debates (Weinstein, 2016; Smialek, 2015). O’Boyle and Scott (2016: 338) see this fragmentation and subsequent resistance to definition as a benefit, helping metal to retain its transgressive edge and avoid 'ossification' by resisting classification. While O'Boyle and Scott (ibid: 341 ) argue that metal will survive and flourish by evolving beyond genre categories, attempting to define and classify genres still has value. As Smialek's (2015: 2964) extensive analysis of existing metal taxonomies shows, metal operates in a core-andperiphery model; there are certain bands or albums that are indisputable and form the core of the genre while the outer boundaries and contentious examples are heavily contested. While the specifics of metal's boundaries remain hard to concretely and discretely define, genre labels and their boundaries are of paramount importance to many metal fans as a primary way of determining an artist's authenticity (Smialek, 2015; Kahn-Harris, 2014). Furthermore, genre labels are a useful way of expediting discussion and analysis of metal, as all categories are, provided that a consensus is achieved on their definition. Establishing a consensus is a key difficulty, as metal genres tend to be defined in terms of which bands or songs are canonically included within a genre rather than by establishing a set of definitive characteristics. $^{1}$

Scholars such as Eric Smialek and Theodore Gracyk have discussed genre in metal in some detail, commenting on the difficulty inherent in the contradictory nature of a variety of fanbased genre taxonomies (Gracyk, 2016). Smialek notes that genre characteristics are closely connected to authenticity and social identity; thus, a thorough knowledge of the musical significations of genres is one of the ways in which metal fans can bolster their position within their communities (2015: 117). He analyses a variety of common genre taxonomies that exist within metal fan communities, noting that metal fans police the accuracy of such taxonomies fiercely (though accuracy is of course subjective to certain communities) and are wary of 'outsider misunderstandings' (ibid: 37). Significantly, extreme metal is defined negatively, by what it is not rather than what characteristics it does demonstrate. Smialek states that these taxonomies all embody some form of narrative relating to the development of the subgenres of metal, noting that an accurate reflection of the way genre operates within metal would become 'absurdly incestuous' and would present additional information that might not be entirely relevant to categorizing genre (ibid: 32-33, 39). Similarly, Gracyk notes that our choice of which fan-based narratives we privilege has implications for the way in which genre is constructed, noting that genre meanings change with time (2016: 777). As time progresses, genre definitions evolve with newer musical expressions that maintain continuity with previous expressions but may no longer fit the exact parameters of a genre label after their evolution. An example of this is the shift in meaning for 'black metal', that initially simply applied to any music that had a Satanic theme (e.g. Venom, Mercyful Fate), yet is now a genre with distinct musical characteristics regardless of lyrical content characterized largely by the 'second wave' of black metal and the infamous Norwegian scene of the 1990s (Smialek, 2015: 30-33) ${ }^{2}$. Gracyk notes that there are problems with this retroactive redefining of genre terms that render metal difficult to define when revisionist fans enter the discourse and de-canonize certain bands, especially with regards to the origins 
of the genre (2016). To show this, Gracyk interrogates Andrew Cope's (2010) narrative of Black Sabbath as the ultimate progenitor of heavy metal to the exclusion of bands such as Led Zeppelin as 'hard rock', arguing that this revisionist exclusion of Led Zeppelin overemphasizes the stylistic unity of heavy metal prior to the NWOBHM movement of the late 1970s (2016: 782-84). Both Gracyk and Smialek argue that musical style alone cannot define heavy metal; in Gracyk's view, there is something about so-called 'hard rock' that early commentators identified as 'heavy metal' despite Cope's differentiation of their musical styles (Gracyk, 2016: 783-84); for Smialek, paratextual elements must also be accounted for, given early definitions of black metal based around lyrical themes and imagery rather than purely musical attributes (2015).

The issues of musical style, genre, and the connection between hard rock and heavy metal have been considered closely by theorists such as Allan Moore and Dietmar Elflein. Moore proposes that hard rock and heavy metal are not two genres but a single genre existing at separate extremes on a continuum (2001: 148). Elflein updates this to include 'extreme metal' as the furthest point on this continuum, as well as indicating the decrease in blues rock influence as one moves from hard rock to extreme metal and the decrease in progressive rock influence if one moves in the opposite direction (2016: 35). While there are undeniable similarities between hard rock and heavy metal, I am yet unconvinced that these models adequately describe the listener's experience of metal music. As indicated in Elflein's version of the model, hard rock has little direct influence on the extreme metal subgenres, except by influencing heavy metal that in turn influences extreme metal. Connecting the two via a continuum shows the historical development of the genres, rather than revealing how metal operates presently. The fundamental issue with this is that it approaches the analysis of metal music from a rock-centric perspective, rather than treating metal as a distinct genre. While metal certainly evolved from rock and might very broadly be considered 'rock music', its systems of pitch organization, timbre, style and expression differ significantly from hard rock to the point that anchoring it back to hard rock in a stylistic model obscures more connections than it reveals. Thus, I am proposing a taxonomy and a model that engage with metal genres from a metal-centric perspective, rather than from a rock perspective.

\section{Categorizing and classifying metal genres}

Beyond understanding the current questions that surround genre in the literature, I wish to propose a means for categorizing the different 'levels' of subgenres in metal. Currently, there is one major division between metal's subgenres within metal studies and fan discourses: the split between heavy metal and extreme metal. The earliest form of this is Weinstein's (2000[1991]: 45-52) distinction between 'lite metal' and 'speed/thrash' metal (though neither category fully encapsulates the current understanding of heavy metal) that is recontextualized by Kahn-Harris is his analysis of extreme metal into its current form of heavy metal versus extreme metal (2007). Extreme metal is usually then understood to fragment into a variety of different subgenres, the most prominent being death metal, black metal, thrash metal, doom metal and grindcore (Kahn-Harris, 2007; Phillipov, 2012; Smialek, 2015). Many of these genres themselves fragment into sub-subgenres and combine with one another or other styles of music. This leads, in some cases, to endless permutations of sub-sub-subgenres that becomes almost farcical. Conversely, heavy metal is generally held to be a single entity, with potential subgenres such as power metal or hair/glam metal rarely being discussed in much detail or indicated in genre models. The models of metal genres provided in the academic literature (e.g. Lilja, 2009: 30) tend to follow the development of heavy metal up to the mid1980 s, with the fragmentation of genres at this point noted but not commented upon. 
Those writing about extreme metal often note the variety of subgenres present in this term, but rarely illustrate the connections and development between them. Smialek's (2015) critique of the various models that exist within fan discourses on metal genre illustrates the benefits and drawbacks of the various approaches taken to categorizing metal subgenres (especially regarding extreme metal), yet he does not propose his own means of categorizing genres. Furthermore, none of the existing models yet reconcile the subgenres of metal that could plausibly be included under both extreme metal and heavy metal (such as folk metal and progressive metal), or the extensive hybridization of genres between metal and non-metal genres. Additionally, there is a recent trend in scholarship that seeks to develop systems to automatically classify metal subgenres, presumably for use with music discovery and sharing software (Tsatsishvili, 2011; Mulder, 2014). These automatic systems note the problems with existing taxonomies (namely their inconsistency and the lack of a consensus regarding definitions), while proposing their own means of identifying genre characteristics that typically rely on one musical element over others (e.g. pitch class space and pitch space in Mulder, 2014). While this research is a viable means of genre categorization, I contend that these taxonomies are less helpful being rooted in a single musical element, whereas the model proposed later in this article incorporates several. Furthermore, these approaches are largely concerned with providing data for use in software, rather than providing salient definitions of subgenres for scholarly use.

Given these shortcomings, I have developed my own taxonomy that is tailored for use in an academic context to develop a common vocabulary of genres and subgenres in metal studies. It reflects the existing literature on metal genres (especially regarding the organization of extreme metal) as well as my own understanding and experiences with genre relationships based on my participation in various metal communities. To this end, I have only included what I consider to be the most prominent subgenres present in the literature and fan communities, excluding most of the individual 'microgenre' labels that exist at the intersection of various subgenres from a simple combination of two or more genre names (e.g. progressive death metal, blackened thrash metal, symphonic black metal, etc.).

Likewise, I have excluded genre labels that predominantly describe lyrical themes rather than differences in musical style (e.g. Christian metal or pirate metal). Table 1 illustrates my proposed taxonomy for metal genre classification. I use four categories: 'genre', meaning the overarching 'umbrella term' for a musical style; 'major subgenre', meaning the dominant subdivisions of the two main genres; 'minor subgenre', meaning those further subdivisions of subgenres; and 'hybrid subgenre', meaning those subgenres that arise not from subdivision, but a combination of various metal and/or non-metal subgenres. In the case of hybrid genres, I have included simple formulae to indicate the genres that comprise them. These are not intended to be definitive but rather guidelines for those who may not be familiar with the basic elements of certain subgenres. A fifth category, 'related genres', is included in the table below to list genres that are closely connected to metal yet are heavily contested or rejected as 'true' metal genres ${ }^{3}$. Their inclusion in this table is to indicate roughly where they align with certain metal genres. Depending on the level of detail desired, one might also include the 'microgenres' as an additional category, but I have found that such specific detail unnecessarily complicates the tables and images used in this article.

These categories are intended to function analogously to taxonomic ranks used in biology (e.g. Kingdom, Phylum, Class, etc.). However, the development of metal subgenres is not as linear as biological evolution, as individual bands frequently and freely combine attributes of numerous subgenres in their music, occasionally creating new subgenres in the process. 
Many of metal's subgenres 'evolved' simultaneously in the mid-to-late 1980s, drawing influences from a variety of sources. Furthermore, the potential for creating new subgenres simply by crossing any number of existing subgenres makes isolating 'pure' instances of a subgenre increasingly difficult. Rather, this taxonomy is meant to aid in classifying metal subgenres by understanding how they relate to one another, organizing them in relation to broader 'umbrella genres' that share core characteristics. This creates the potential to then connect individual bands back to these various categories depending on their demonstrated musical style and belonging within a genre. Scholars may then draw connections between various bands at their various levels of influence and style to further understand how musical decisions are made and musical style is developed. For example, we might consider Dimmu Borgir a 'symphonic black metal' band (Smialek, 2015). Although this subgenre is not on the taxonomy, we can work backward from two points: black metal and symphonic metal. Dimmu Borgir displays many of the categories of black metal in the use of harsh vocals, darker modes, tremolo-picked black metal riffs (especially on their earlier albums) and black metal aesthetics including a Satanic lyrical theme and corpsepaint when performing live. Their symphonic metal characteristics include orchestral instruments and arrangements, an emphasis on keyboard melodies and triadic harmonies, a clearer production style and the adoption of a Romantic aesthetic in elements of their visual media (Smialek, 2015). We might then trace these influences back through the taxonomy, understanding a 'stylistic lineage' from heavy metal, to power metal, to symphonic metal, to Dimmu Borgir alongside a lineage from extreme metal, to black metal, to Dimmu Borgir (via symphonic black metal if one included microgenres). This reveals something intriguing about the genres of heavy metal and extreme metal: elements from heavy metal subgenres can 'cross' the divide into extreme metal but the reverse is not true. That is to say, in spite of the symphonic metal influences, Dimmu Borgir remain an extreme metal band, operating within black metal, while symphonic black metal functions as a microgenre of black metal. Likewise, melodic death metal, with its extensive NWOBHM influence (Smialek, 2015; Hillier, 2018) remains a subgenre of death metal. The hybrid subgenres are not considered 'blackened symphonic metal' or 'deathened heavy metal' (connoting that they are foremost heavy metal subgenres which have been modified) but 'symphonic black' and 'melodic death' (connoting that they are foremost extreme metal subgenres which have been modified).This suggests that, on some level, extreme metal influences 'overwrite' heavy metal influences in a band, meaning that bands who draw influences from both heavy metal subgenres and extreme metal subgenres are ultimately classified within extreme metal. While the full investigation of this phenomenon is beyond the scope of this article, future work may examine how and why metal communities follow these classifications and if there are exceptions.

Figures 1, 2, and 3 demonstrate the connections between these subgenres in graphic form to complement the taxonomy shown in Table 1. These images illustrate the 'levels' or 'ranks' of subgenres via arrows that illustrate how the genres and subgenres relate to one another, with the arrows representing 'divides in to' (e.g. extreme metal 'divides in to' death metal which 'divides in to' melodic death metal, etc.). Resultantly, it is unsurprising that these levels are flexible: for example, a hybrid subgenre such as grindcore is on the same 'level' as other major subgenres such as death metal, black metal etc. While it isn't a direct subdivision of extreme metal, it is still a prominent genre that itself divides down into other hybrid and minor subgenres. Thus, this taxonomy is not meant to show the evolution of metal's myriad subgenres (although some of these relationships are naturally reflected by considering subgenres as divisions of a parent genre) and is distinct from other lineage-based models. 
Table 1: A Taxonomy of metal subgenres

\begin{tabular}{|c|c|c|}
\hline Genre: & HEAVY METAL (HM) & EXTREME METAL (EM) \\
\hline $\begin{array}{l}\text { Major } \\
\text { Subgenre: }\end{array}$ & $\begin{array}{l}\text { Power metal } \\
\text { Hair/Glam metal } \\
\text { Traditional heavy metal }\end{array}$ & $\begin{array}{l}\text { Death metal } \\
\text { Black metal } \\
\text { Thrash metal } \\
\text { Doom metal } \\
{\text { (Grindcore) })^{4}}\end{array}$ \\
\hline $\begin{array}{l}\text { Minor } \\
\text { Subgenre: }\end{array}$ & $\begin{array}{l}\text { NWOBHM } \\
\text { US Power Metal (USPM) } \\
\text { European Power Metal } \\
\quad \text { (EUPM) } \\
\text { Traditional Doom }{ }^{5}\end{array}$ & $\begin{array}{l}\text { Melodic Death Metal } \\
\text { Technical Death Metal } \\
\text { Atmospheric Black Metal } \\
\text { Groove metal } \\
\text { Stoner Doom } \\
\text { Funeral Doom } \\
\text { Drone }\end{array}$ \\
\hline $\begin{array}{l}\text { Hybrid } \\
\text { Subgenre: }\end{array}$ & $\begin{array}{l}\text { Folk metal [HM }+ \text { folk] } \\
\text { Progressive metal [HM }+ \\
\text { Progressive rock] } \\
\text { Symphonic metal [Power metal } \\
\quad+\text { classical/orchestral] } \\
\text { Gothic metal } \\
\text { [symphonic/power }+ \\
\text { doom] }\end{array}$ & $\begin{array}{l}\text { (Extreme) Folk metal [EM + folk] } \\
\text { (Extreme) Progressive Metal [EM + } \\
\text { Progressive rock] } \\
\text { Avant-garde/Experimental Metal } \\
\text { Sludge [doom + hardcore] } \\
\text { Grindcore [death/thrash + hardcore] } \\
\text { Slam [death + hardcore punk) } \\
\text { Crossover thrash [thrash + hardcore punk] } \\
\text { War Metal (AKA 'bestial black metal') } \\
\quad \text { [death + black + grind] } \\
\text { Depressive/Suicidal Black Metal (DSBM, } \\
\text { AKA 'Cascadian Black Metal') } \\
\quad \text { [black + doom] } \\
\text { Viking metal }{ }^{6} \text { [a specific version of black }+ \\
\text { folk] }\end{array}$ \\
\hline $\begin{array}{l}\text { Related } \\
\text { genres }\end{array}$ & $\begin{array}{l}\text { Alternative metal [alternative } \\
\text { rock }+ \text { metal } \\
\text { influences] } \\
\mathrm{Nu} \text { metal [alternative rock }+ \\
\text { rap/hip-hop }+ \text { metal } \\
\text { influences] } \\
\text { Industrial metal [industrial }+ \\
\text { alternative metal] }\end{array}$ & $\begin{array}{l}\text { Metalcore [melodic death metal + hardcore } \\
+ \text { crossover thrash] } \\
\text { Deathcore [death metal }+ \\
\text { hardcore/metalcore] }\end{array}$ \\
\hline
\end{tabular}




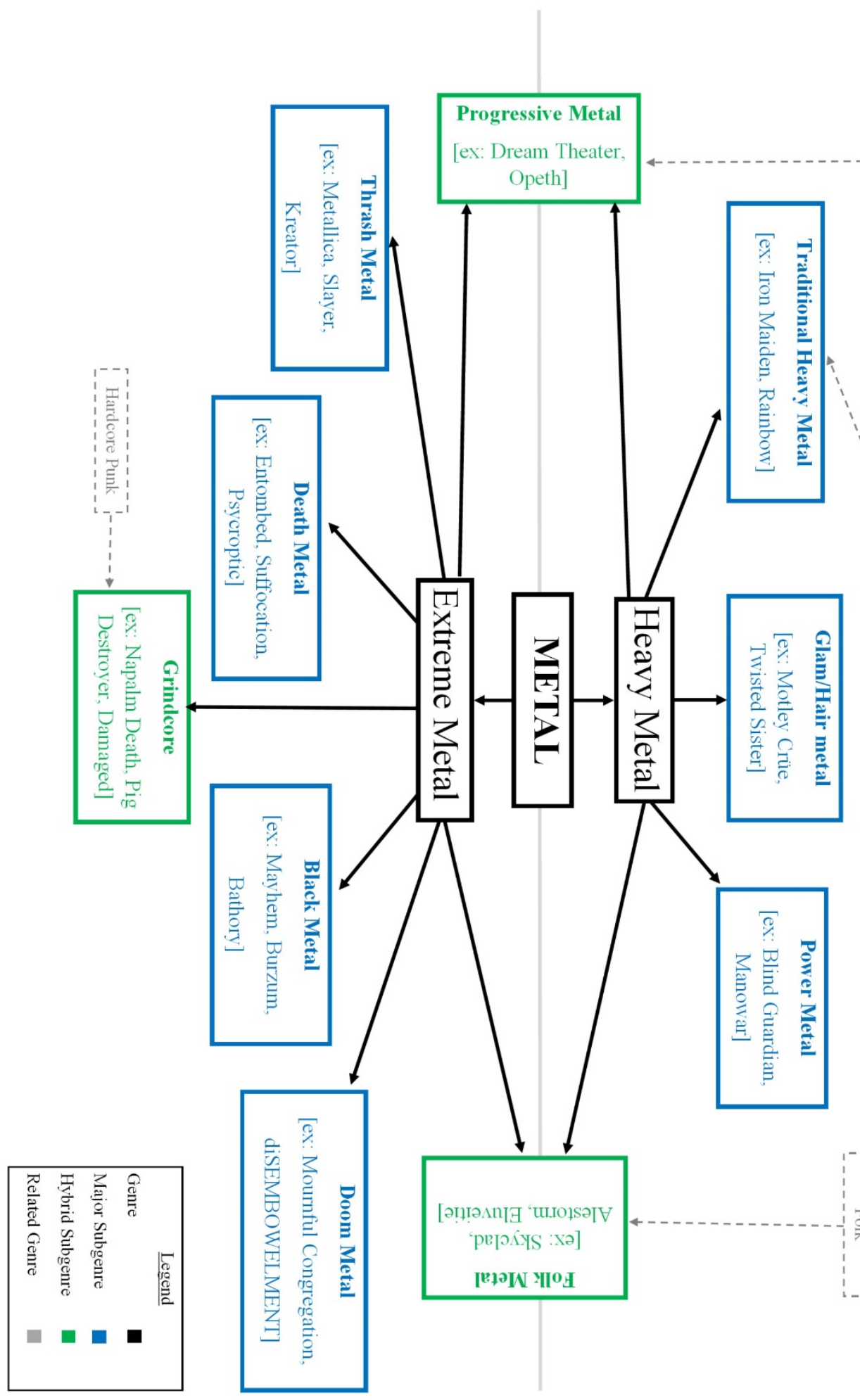

Figure 1: Major subgenres of metal ${ }^{7}$ 


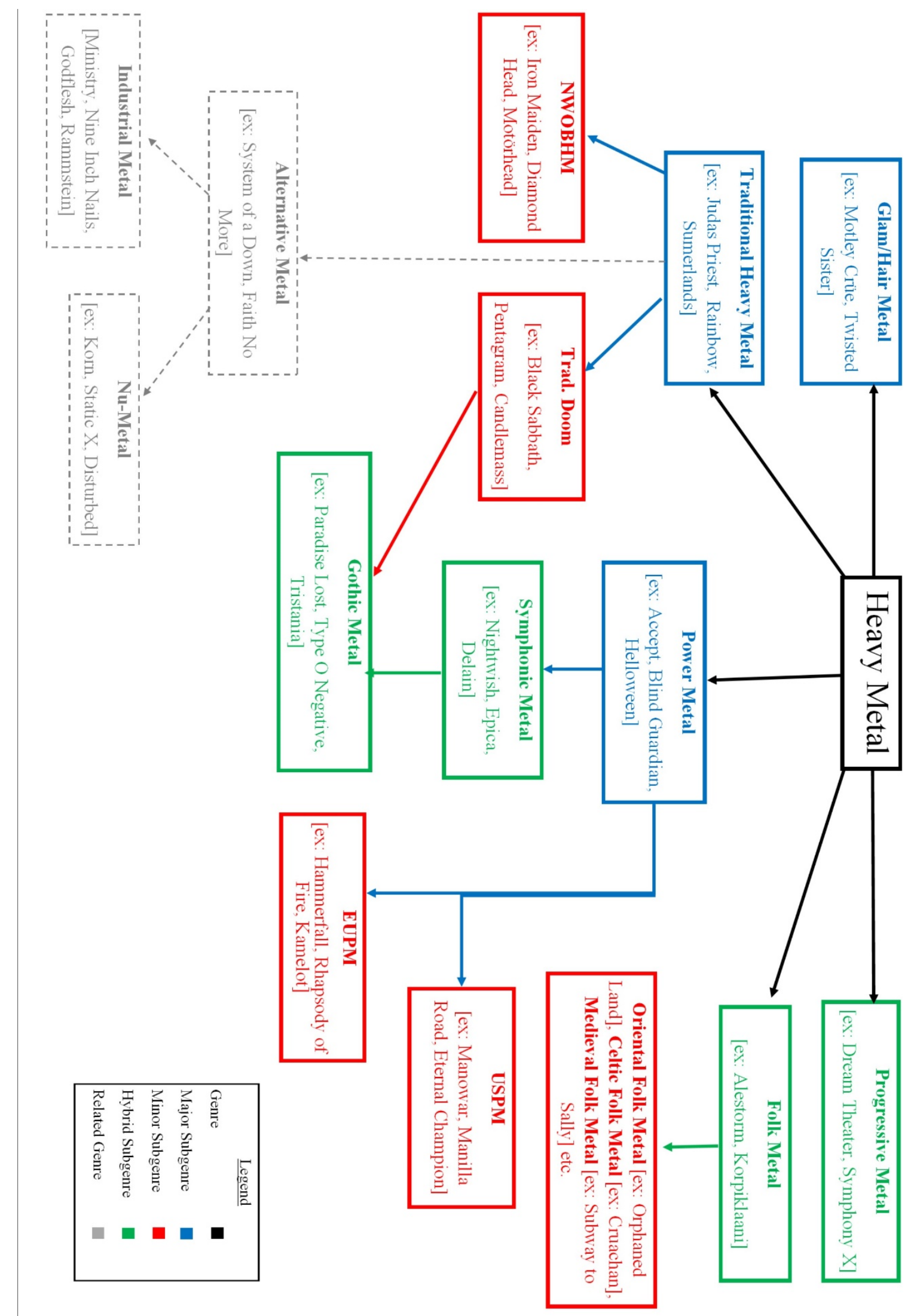

Figure 2: Heavy metal subgenres 


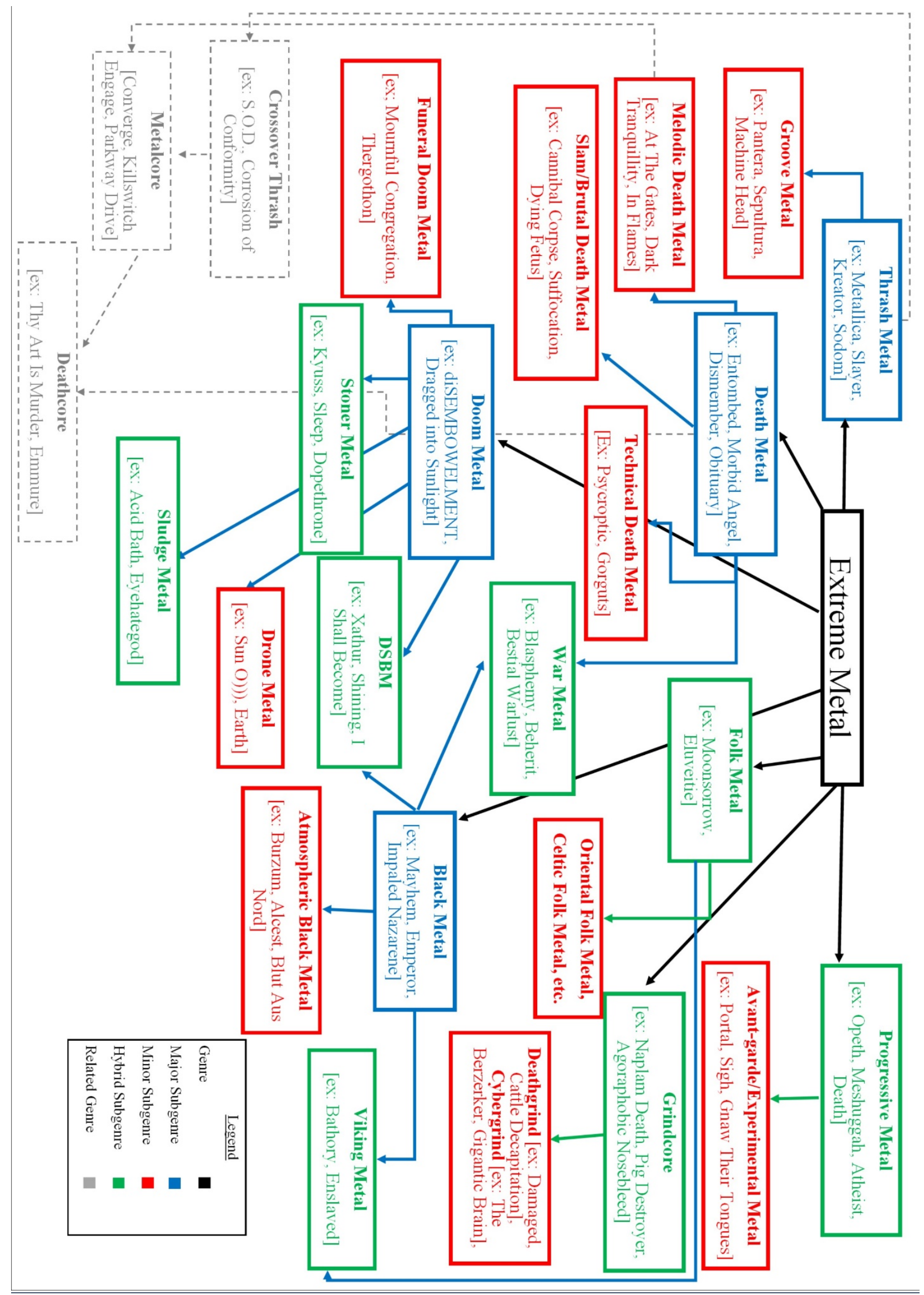

Figure 3: Extreme metal subgenres 
As mentioned above, a core element of this taxonomy is that it categorizes genres primarily around discrete differences in musical style, rather than a consideration for lyrical or aesthetic elements. This is somewhat contentious within metal communities, as many subgenres are used in the discourse that are derived from lyrical themes (e.g. pirate metal, Christian metal, Viking Metal). There is also an element of 'elitism' associated with policing genre labels based on lyrical themes within metal communities, given that correctly identifying genre labels for a band is a means of establishing subcultural capital within metal communities. Listening to a wide variety of music from different subgenres to distinguish between the minutiae of their differences takes time, effort, and expertise, hence the conferral of subcultural capital on one who can do this 'correctly'. Conversely, looking at an album cover or reading a lyric sheet to establish an aesthetic theme for a band is held to require comparatively less engagement, effort, and expertise, connoting a surface-level fan of the music who will 'incorrectly' identify a given bands subgenre. This is further complicated by the polysemous use of genre labels within metal communities, where genre labels have two divergent meanings that are equally intelligible to those familiar with the subject. To illustrate, the phrase 'black metal that sounds like Taylor Swift' makes little sense; Taylor Swift does not perform black metal, nor would anyone reasonably mistake her musical style with that of black metal. However, the phrases 'Viking metal that sounds like Amon Amarth' and 'Viking metal that sounds like Bathory' both make sense as phrases, even though Amon Amarth (a melodic death metal band) and Bathory (a black metal band) bear little musical similarity beyond both being metal bands. While a fan of Amon Amarth-style Viking metal might not enjoy Bathory-style Viking metal (and vice versa), one who is familiar with both bands understands that the first reference is to the lyrical/aesthetic theme and the second is to the musical style, even if they might disagree with the usage of the genre label. Due to this polysemy, I believe it is unlikely that theme-based genre labels will decline in usage within metal fan communities as they do not significantly impede understanding or communication even though certain groups of fans disagree with their usage.

That said, polysemy raises some complications when categorizing metal genres and understanding their relationships to one another; which meaning of Viking metal do we understand as the default and which do we understand as an aberration, especially as there are examples of literature that use both (Spracklen, 2015; Hoad, 2012). Furthermore, classifying bands based on lyrical themes alone has the potential to obscure more connections that it reveals. For example, Alestorm and Swashbuckled may both, broadly, be considered 'pirate metal'; they sing about pirate mythology and prominently feature pirate paratexts and aesthetic elements in their performance and songwriting. Yet these two differ significantly in their musical style; Alestorm are musically a folk metal band, owing to their liberal usage of folk instruments (accordion, fiddle etc.) and marked musical influence from folk tunes alongside a broad power metal sound driven by up-tempo riffs and guitar harmonies. Swashbuckled, however, are musically death metal, lacking the levity and folk influence of Alestorm and featuring the harsh vocals, down-tuned guitars and darker modalities common to death metal. This raises the question that, if there is no musical continuity between bands within a proposed musical genre, does the genre label 'Pirate metal' have any meaning? If Swashbuckled and Alestorm do not bear significant musical similarities (beyond both broadly being metal bands), then what is the point of using the pirate metal label to describe both bands other than establishing that they both have a pirate aesthetic? Such things can be communicated by using phrases in the vein of 'pirate-themed folk metal' or 'Viking-themed melodic death metal'. 
Considering these complications, I propose that elements of musical style should be the primary, defining characteristics of a metal subgenre within metal studies. Whilst aesthetic and paratextual elements are important to consider, they are paratextual, with musical elements as the main 'text' of metal. The problems with lineage-based definitions of bands and subgenres are evident in that they are subjectively based on who is making the definitions and are liable to change with time (Gracyk, 2016). The problems with theme-based labels have been outlined above. This is not to say that all metal studies must be co-opted by musicology or music analysis, nor that there is no value in studying the non-musical elements of metal's genres and how they inform meaning; certainly, these attributes and methods of inquiry still have value. I agree with both Smialek and Gracyk when they observe that musical style alone demonstrably cannot define a metal subgenre. However, when it comes to definitions of musical subgenres, they must remain rooted in musical attributes, with aesthetic elements a secondary consideration. Ideally, basing genre definitions on musical style will incorporate an element of objectivity into these definitions (insofar as defining and organizing musical styles can be objective) and address the shortcomings of other methods. These definitions should still be flexible, grounded in musical evidence, and it is likely that they will also evolve in time as the music evolves.

\section{A model for classifying and understanding the attributes of metal subgenres}

Given the need to define metal genres based on their musical characteristics, I have also developed a model to assist with this that suits the multidisciplinary nature of metal studies. My model is inspired by Moore's four-layered model for analyzing rock music that consists of high-note melody, low note melody, harmony, and rhythm (Moore, 2001: 33-34).

Previously, I adjusted this model to better fit the specific characteristics of metal genres, using four layers of melodic/voice leading, harmonic/chordal, rhythm and aesthetics (Hillier, 2017). Notably, both models are specifically used for musical analysis: Moore's model is almost entirely focused around harmony, with rhythm being discussed where relevant to its harmonic implications. My own earlier model found it necessary to also incorporate aesthetic elements due to their importance within metal but was again oriented around harmonic analysis. The current model seeks to move beyond this narrow single usage without losing its usefulness for musical analysis.

Figure 4 illustrates the model, the parameters and their connections. The two main goals of the model are to outline the specific ways in which these layers define subgenres and to provide a framework through which metal analysis might be conducted. Extensive analyses that come under the purview of each of the five layers already exist, therefore this model is attempting to codify the connections between these characteristics and synthesize the themes that are present in the broad literature, addressing the need for theories, models and methodologies in metal studies (Weinstein 2016). Significantly, it is the intersections between these layers that often reveal the most information about genre characteristics and it is at these intersections that 'genre' itself emerges. In this sense, the model is multi-functional and relies on a degree of perception and phenomenology; all five layers occur simultaneously, yet our perception of any given musical moment may cause certain elements or interactions to foreground themselves in our mind whilst the others remain present and active in the background ${ }^{8}$. Given that metal is first and foremost a genre of music, the model is predominantly intended to assist with the analysis and discussion of musical objects songs, performances, albums etc. While it is not focused on cultural elements, its dimensions do allow for cultural elements to be connected to musical elements (and vice versa) which is 
an essential part of any analysis that seeks to understanding how meaning ${ }^{9}$ is derived and disseminated in metal.

\section{Pitch}

Pitch refers to musical tones and their interactions: harmony, voice-leading, melodic note choices, modality, tonality etc. Of particular importance to metal is the impact of distortion on the aural quality of chords and the implications of this for vertical and horizontal harmony, with extensive scholarship on the connection between the two (Walser, 1993; Lilja, 2004; 2009; Herbst, 2018). The role of pitch in a given song has implications for genre, and many metal genres characteristically make use of certain modalities or are regarded as being more consonant or dissonant than others (Hillier, 2018; Lilja, 2009; Purcell, 2003). This layer has an especially close connection with timbre and aesthetics. (A)tonality and modality can be used to support a certain aesthetic, with extreme metal genres typically tending toward more chromatic and aurally jarring sonorities alongside other musical choices in an attempt to confront and disorient their listeners (Phillipov, 2012). Much musicology (and specifically music analysis) on metal music unsurprisingly focuses on pitch, given that harmonic analysis forms the bedrock of much traditional music analysis. Recent scholarship has illustrated the need to move beyond a solely pitch-focused approach to metal to incorporate a wide variety of different factors and analytical methods, especially regarding extreme metal (Scotto, 2016; Smialek, 2015). While there are certainly analyses that demonstrate that heavily-adapted versions of traditional musicological methods (e.g. Roman Numeral analysis Schenkerian analysis) have some use (Lilja, 2009), this tends to mainly be restricted to 'classic' heavy metal, with extreme metal subgenres resisting analysis through these methods because of their divergent approaches to pitch material. Consequently, the analysis of pitch material in these subgenres should be connected to other musical factors.

\section{Timbre}

Timbre refers to the specific 'tone colour' or sonic qualities of various instruments in music. In metal, distortion is the foundational timbre around which much of the genre's meaning is structured; many authors have noted that distortion is central to the experience of fans and musicians (Lilja, 2009; Mynett, 2016; Walser, 1993). Resultantly, the use of timbre is one means by which genre can be signified, as different genres can have different 'degrees' of distortion or may utilize different non-distorted timbres (for example, the inclusion of orchestral instruments in symphonic metal). While these differences may seem subtle, recent scholarship illustrates that their differences can be quite complex and can confer layers of meaning with regard to genre (Herbst, Reuter and Czedik-Evsenberg, 2018; Pillsbury, 2006; Scotto, 2016). Perhaps the clearest impact of timbre on genre is the use of harsh vocals (i.e. screaming, growling etc.) versus conventionally sung vocals. In extreme metal genres, harsh vocals are not only a deliberate genre characteristic but are pleasurable experiences for the listeners of such genres, requiring a reorientation of listening practices (Phillipov, 2012). Timbre and aesthetics are especially intertwined in metal music, as timbral changes often occur alongside other extra-musical aesthetic changes (Smialek, 2015; Hillier, 2018). A particular element of the musical experience that arises from this connection is the role of production style, itself a means of differentiating genres (Hillier, 2018; Mynett, 2016; Smialek, 2015). It sits somewhere in the middle of the two layers; often, aesthetic choices and values drive the desire to achieve certain production values, yet timbre is often how these production choices affect the aesthetics. This means that production styles occur at an 
intersection of these layers. Recent scholarship has also suggested that 'heaviness' arises out of an intersection between elements of timbre, pitch, and aesthetics (Herbst, 2018).

\section{Form}

Form refers to the structure and organization of musical material in a song. While several aspects of metal's formal organization are derived from broad principles applicable to rock music, it also has many characteristics unique to its subgenres (Elflein, 2016; Schumann, 2015). Extreme metal subgenres are often described as being structurally complex in their organization of musical material, while metal songs that do follow more conventional song structures often have unique variations on them or utilize these conventional structures as a point of difference (Elflein, 2016; Purcell, 2003; Smialek, 2015). For example, within death metal subgenres, Entombed's 'Left Hand Path' can be classified as through-composed. While the initial section of the song until 2:10 can be loosely understood as a Verse-Chorus form, the remainder of the song progresses through various riffs and sections without repeating before ending on an extended solo section from 3:50-6:39 that is based around a repeated bassline or chord progression rather than a guitar riff per se and does not neatly fit any category apart from an instrumental outro/solo. Conversely, At the Gates' 'Blinded by Fear', an example of melodic death metal features a conventional Verse-Chorus structure throughout the entire song with each riff splitting neatly into 4-8 bar sections. This difference in form differentiates the genres of (Swedish) death metal and melodic death metal, with the Verse-Chorus structure found in At the Gates' melodic death metal showing an influence from NWOBHM (Hillier, 2017).

Given that riffs are the dominant 'building block' of a song in many metal genres, the organization and characteristics of riffs in a song is a further marker of genre within the broad label of metal (Elflein, 2016). However, other subgenres, such as melodic death metal, differentiate themselves through a lack of riffs, instead utilizing chord progressions (Smialek, 2015). Thus, form and the organization of riffs is an important aspect of classifying genre.

Form intersects prominently with each of the other layers. Harmony and tonality are common means of organizing musical material in metal, as with most music, with particular implications regarding the interaction between riffs, chord progressions, harmony and tonality. As detailed below, rhythm is also a significant aspect in determining the structure of metal riffs, as are timbral and aesthetic changes (Berger, 1997; Elflein 2016; Hillier 2018; Smialek 2015). Consequently, form provides a useful means of relating these other layers to one another within the context of a piece of music, whilst also contributing to genre definitions in its own right.

\section{Rhythm}

Rhythm refers to a broad range of characteristics such as tempo, meter, pulse, and beat, as well as the interaction between these characteristics and their organization in a given piece of music. Metal riffs are frequently structured around pulses rather than a meter (Elflein, 2010; Walser, 1993: 49) While transcribed riffs may often be represented within a given meter (e.g. 4/4), this is not to say that these riffs must be organized within a meter; indeed, Elflein argues that riffs should be subdivided into groups of pulses for analysis rather than grouped by metered bars as much music analysis would do, as this reveals the smallest units of metal's musical language $(2013)^{10}$. This makes sense of cases where metal riffs do not fit within metric organization, such Iron Maiden's 'Number of the Beast' (Elflein, 2010: 286). Genre and rhythm interact via the conventions of riff writing and pulse organization; genres falling 
on the more extreme end of the metal spectrum tend to utilize independent groupings of pulses while less extreme genres utilize pulse organizations more closely aligned with blues music, such as the $3+3+2$ grouping (ibid: 238 ). Tempo is also a marker of genre, with genres like doom metal being characterized in part by slower tempos. Given the established importance of pulse organization on riff structures, rhythm and form are closely connected. Likewise, rhythm and pitch are closely connected, as rhythmic accents can foreground key pitches to the listener whilst faster tempos can result in pitches being obscured due to the speed of their succession, requiring an adaptation of conventional pitch analysis methods (Lucas, 2018).

\section{Aesthetics}

Aesthetics refers to the musical and extra-musical characteristics of a piece of music that are not covered by the previous categories. Of particular importance are the extra-musical 'paratexts' that exist alongside the musical material, such as artwork, band names and logos, lyrics, promotional material etc. It is important to note that these paratexts are not isolated from musical choices but are closely connected to them; as mentioned earlier, aesthetic changes often occur alongside musical developments in a genre (Hillier, 2018; Smialek 2015). Aesthetics are integral to defining and presenting oneself as authentically metal and are often the clearest markers of genre, especially to those unfamiliar with metal (Weinstein, 2000). Thus, while the aesthetic layer is closely connected to the other layers of the model, it is something of a complicated relationship. While these stereotypes often signify genre, it is important to keep in mind that they do not necessarily cause genre. That is to say death metal does not have to focus lyrically on topics of death and gore, nor do black metal musicians have to perform in the 'corpsepaint' make-up often associated with the genre. In this sense, aesthetic elements may indicate genre without defining it. However, it is undeniable that aesthetic elements represent musical changes, especially for fans who are less familiar with the detailed musical characteristics that comprise different subgenres (Smialek, 2015). Additionally, aesthetic elements are often integral to the atmosphere or 'feel' of a certain genre and prominently interact with the other layers in this capacity. For example, if one were to take the introductory riff to Slayer's 'Raining Blood' [0:33-0:44] and play it in a major key (pitch) with a clean guitar tone (timbre) and a swung rhythm (rhythm) would be difficult to identify it as 'metal' without some significant aesthetic context (e.g., that this was a contrasting section to the main song that is more conventionally metal, clarified by the aesthetic elements, structure, or paratexts) or foreknowledge of what the original riff was. Thus, aesthetics warrant consideration as a key element of defining and especially communicating genre. 


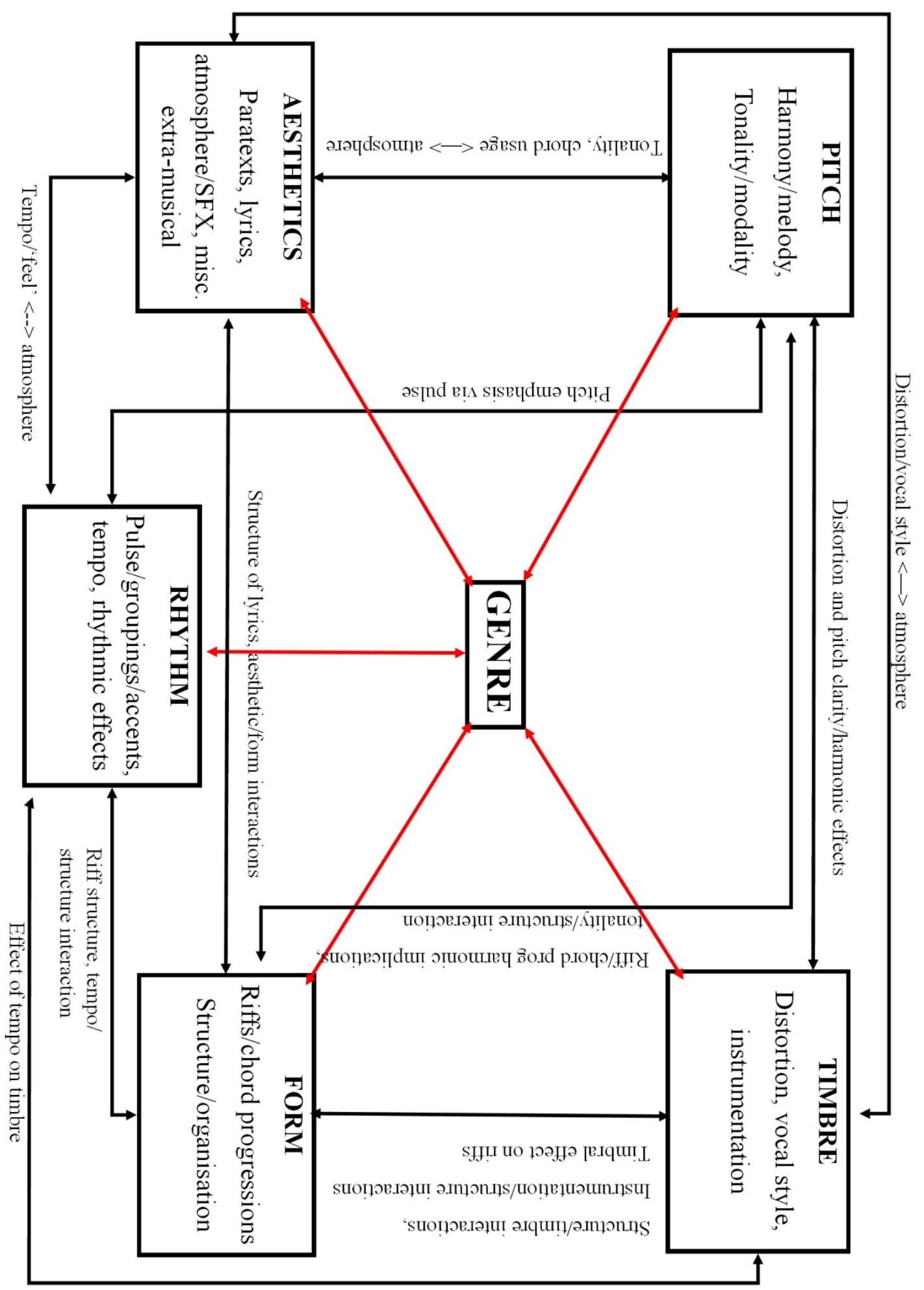

Figure 4: Proposed model for metal genre classification 
There are two main ways to apply the model: from the perspective of an individual artist and from the perspective of a genre. ${ }^{11}$ Applying the model on a band-by-band basis can illustrate how permeable genre boundaries in heavy metal are, as there are many bands that fit within multiple subgenres, the specific relationships between which become evident when examining the elements of genre together. For example, In Flames combine elements of death metal and NWOBHM into new forms as the model can illustrate. Examining their use of pitch, In Flames draw upon minor modes in their chords and riffs, tending to be more consonant than most death metal bands while retaining occasional dissonances or chromatic movements. (Hillier, 2017; Smialek, 2015). Melodies are clearly heard within the mix and are often harmonized in thirds as seen in NWOBHM (Smialek 2015: 197). Timbral elements illustrate a middle-ground between death metal and NWOBHM; while the harsh vocals used in their work are a marked influence from death metal, the intensity guitar distortion sits somewhere between death metal and NWOBHM, though it is still derived from the classic Swedish death metal tone (Hillier, 2018). A relatively clean and clear production style is an influence from NWOBHM (Smialek, 2015). Their early work in 1995-1996 also displays a common instrumentation with NWOBHM where the bass guitar is used in a higher register and a melodic role, though this would disappear in later years (Hillier, 2018). Structurally, In Flames are notable in widely using Verse-Chorus forms more so than many death metal bands (Smialek, 2015). There is a mixture of riff-based and chord-progression based composition within their 1990s albums such as The Jester Race, again striking a balance between their death metal and NWOBHM influences (Hillier, 2017).With regard to rhythm, In Flames often use syncopated and speech-like vocal patterns while their rhythm-guitar power chords tend to be slower than other extreme metal subgenres in order to foreground the emphasized melodic elements (Smialek, 2015: 199-201). Occasional influences from Swedish folk music can be seen in their usage of a hemiola and triple time meters in 'Moonshield' among other songs (Hillier, 2017). The aesthetic elements represent the greatest change from both death metal and NWOBHM: lyrics become melancholy and dour (as opposed to death metal's focus on gore and violence) yet the album art and logo associated with early In Flames remains a sharp-edged, red logo that aesthetically aligns with other death metal bands. Later logos (such as those on Whoreacle and Colony) become much more sterile and futuristic in the vein of other melodic death metal bands (Hillier, 2018: 1418). Most notably, in spite of the heavy influence from NWOBHM illustrated above, In Flames still considered themselves a death metal band, as did the other Gothenburg bands during this period, later being labelled 'melodic death metal' (Smialek, 2015; Hillier, 2017). As such, we are able to use the features revealed by the model to understand how In Flames represent melodic death metal and establish a basis by which we might categorize many other bands as the same.

The model can also be applied in reverse, taking an established genre label as a starting point to illustrate, define, and codify genre characteristics. In this case, I will use the model to clarify the genre characteristics of traditional heavy metal with reference to established bands within this subgenre such as Iron Maiden, Judas Priest, and Black Sabbath.

With regard to pitch, the harmonic features of traditional heavy metal are well established. Riffs are modal (particularly after the NWOBHM) with marked influences from blues (prominently in Black Sabbath's early music), hard rock, and Romantic chord progressions and voice leading styles (Lilja, 2009). Harmonized lead guitar lines, typically in diatonic thirds, are a distinct feature of the songwriting of bands such as Iron Maiden and Judas Priest 
and have widely influenced most metal subgenres. Vertical harmony tends to be fairly consonant, based around power chords or major chords accentuated by the distortion characteristic of the subgenre. Guitar riffs tend to remain in the standard register for a guitar while vocals in traditional heavy metal are usually in a high male register exemplified by vocalists such as Bruce Dickinson and Rob Halford.

With regard to rhythm, some earlier traditional heavy metal retains the metric organization common to hard rock while some is organized around pulses, such as Iron Maiden's 'Number of the Beast' (Elflein, 2010). Relative to other metal subgenres, heavy metal tends to be midtempo with marked variations. Black Sabbath, for example, exhibit the slow tempos that would later be codified into doom metal (e.g. the songs 'Iron Man' and 'Hand of Doom') while those traditional heavy metal bands leaning closer to 'speed metal' (e.g Motorhead) are considerably faster. Certain rhythms, such as the two semiquaver-quaver 'gallop rhythm' associated especially with Iron Maiden, are characteristic of the genre as well (Elflein, 2010).

With regard to timbre, distortion is the main feature. Relative to most rock music, the distorted timbre in traditional heavy metal is considerably heavier, yet relative to most extreme metal subgenres (e.g. death metal, black metal) it is somewhat milder. Harsh vocals are very uncommon in traditional heavy metal, with the standard vocal style being a higherregister semi-operatic vocal style exemplified by the aforementioned vocalists. Timbre and pitch are closely connected in traditional heavy metal, with the slightly less-distorted timbre relative to extreme metal facilitating more triadic chords and perhaps influencing the use of chord progressions and semi-functional harmony in traditional heavy metal relative to riffs that have less variation in harmonic role in extreme metal (Lilja, 2009: 117-122).

Additionally, the dissonance generated by distorted minor triads is avoided in traditional heavy metal where some extreme metal genres embrace it more fully (Hillier, 2018: 13).

With regard to form, traditional heavy metal is based primarily around riffs more than other means of song organization (Elflein 2016). Other methods of song organization common to hard rock and blues are still present in traditional heavy metal, yet the song structures are somewhat more complex (Ibid). Focusing mainly on Judas Priest and Black Sabbath, Elflein demonstrates that, while the vocal structures of traditional heavy metal still outline a broad Verse-Chorus structure, the microstructure of riffs and pulses within these broader structures can be extremely complex, thus evolving the structures of blues and rock into new heavy metal forms (Ibid: 40-47) ${ }^{12}$. As discussed earlier, these structural elements in traditional heavy metal are also found in genres influenced by traditional heavy metal such as melodic death metal (Hillier, 2017; Smialek, 2015: 195-7, 232-4), suggesting that they are a key marker of the genre.

With regard to aesthetics, traditional heavy metal has a clearly defined 'visual code' that has been analyzed at length (Weinstein, 2000). The visual aesthetic of performers is a combination of general 1970s and 1980s rock aesthetics (long hair, blue jeans and other working-class symbols) alongside a biker aesthetic of black leather and motorbikes prominently introduced by Judas Priest (Ibid). Lyrically, Deena Weinstein's 'Dionysian and Chaotic' lyric themes are likewise well known (ibid) while the visual elements display darker themes such as the occult (e.g. the witch on the Black Sabbath album cover) and madness (e.g. Iron Maiden's Piece of Mind).

These examples are not intended to be definitive of certain subgenres but to illustrate how one might begin to define genres by using the model to reveal connections between the 
various musical and extra-musical elements that comprise metal songs, bands, and genres. The traditional heavy metal example reveals one of the striking elements of the genre: the variation within the parameters. Given that many traditional heavy metal bands were forerunners in codifying the genre and its constituent features, it is unsurprising that there are variations in many of these areas (especially rhythm and macro-structure) and this supports Gracyk's argument that there was little stylistic unity prior to the NWOBHM movement (2016: 782-84). What is most interesting are the areas where there is little variation (e.g. timbre, aesthetics, harmony, and riff-based structures), suggesting that these elements codified early on in the development of heavy metal and are perhaps the elements of the genre that have persisted into its contemporary expressions. It is these relationships that the model hopes to reveal.

\section{$\underline{\text { Conclusion }}$}

Given the importance of genre to metal music, fans and, academics, the model and taxonomy proposed in this article address a gap in the field by unifying the many characteristics that have been discussed by scholars. This is a step towards establishing a consistent vocabulary and methodology within metal studies for discussing, analyzing, and classifying metal music and its various subgenres. While the model proposes the elements that should contribute to genre definitions, establishing the specifics of these definitions would require significantly more detailed and comprehensive analysis that is beyond the scope of this article. Further research may use this model in case studies that can establish the key features of metal genres beyond the shallow and often contradictory definitions that are currently used by fans and scholars. The taxonomy proposed in this article further assists scholars in developing an understanding of the relationships between various metal genres and bands that operate within numerous subgenres. From this, future work might establish a better understanding of how these characteristics and relationships are manipulated in the creation of musical identities in metal.

\section{$\underline{\text { References }}$}

Brown, A., Spracklen, K., Kahn-Harris, K. and Scott, N. (2016), 'Introduction: Global Metal Music and Culture and Metal Studies' in K. Spracklen, A. Brown, K. Kahn-Harris, N.W.R. Scott (eds.), Global Metal Music and Culture: Current Directions in Metal Studies, New York: Routledge. pp. 1-21.

Berger, H. (1997), 'The Practice of Perception: Multi-Functionality and Time in the Musical Experiences of a Heavy Metal Drummer,' Ethnomusicology, 41: 3, pp. 464-88.

Cope, A. (2010), Black Sabbath and the Rise of Heavy Metal Music, Ashgate: Farnham, Surrey.

Elflein, D. (2010), 'Slaying the Pulse: Rhythmic Organization and Rhythmic Interplay within Heavy Metal', in N.W.R Scott and I. Von Helden (eds.) The Metal Void: First Gatherings, Oxford: Inter-Disciplinary Press.

(2013), 'Overcome the Pain: Rhythmic Transgression in Heavy Metal Music', in J. H. Hoogstad and B. S. Pedersen (eds.) Off Beat: Pluralizing Rhythm (pp. 71-88): Brill: Rodopi, pp. 71-88. 
(2016), "Iron and Steel: Forging Heavy Metal's Song Structures or the Impact of Black Sabbath and Judas Priest on Metal's Musical Language." Studies' in K. Spracklen, A. Brown, K. Kahn-Harris, N.W.R. Scott (eds.), Global Metal Music and Culture: Current Directions in Metal Studies, New York: Routledge, pp. 35-49.

Gracyk, T. (1996), Rhythm and Noise: An Aesthetics of Rock, Durnham: Duke University Press.

(2016), 'Heavy Metal: Genre? Style? Subculture?', Philosophy Compass, 11: 12, pp. 775-85. http://dx.doi.org/https://doi.org/10.1111/phc3.12386

Herbst, J. (2018), 'Heaviness and the Electric Guitar: Considering the Interaction between Distortion and Harmonic Structures', Metal Music Studies 4: 1, pp. 95-113, http://dx.doi.org/10.1386/mms.4.1.95 1.

Herbst, J., Reuter, C. and Czedik-Evsenberg, I. (2018), 'Guitar profiling technology in metal music production: public reception, capability, consequence and perspectives', Metal Music Studies, 4: 3, pp. 481-506, https://doi.org/10.1386/mms.4.3.481_1.

Hillier, B. (2018), 'The Aesthetic-Sonic Shift of Melodic Death Metal', Metal Music Studies 4: 1, pp. 5-23, doi: 10.1386/mms.4.1.5_1.

(2017), 'Principles of harmony, voice leading and aesthetics in early Melodic Death Metal', Honours Thesis, Sydney: University of New South Wales.

Hoad, C. (2012), “'Hold the heathen hammer high": Viking metal from the local to the global', In: Wilson, O. and Attfield, S. (Eds), Shifting Sounds: Musical Flow: A Collection of Papers from the 2012 IASPM Australia/New Zealand Conference. Dunedin, New Zealand: International Association for the Study of Popular Music, pp. 62-70.

Kahn-Harris, K. (2007), Extreme Metal: Music and Culture on the Edge, Oxford: Berg. (2011), 'Metal Studies: Intellectual Fragmentation or Organic Intellectualism?', Journal for Cultural Research 15: 3 pp. 251-53. http://dx.doi.org/10.1080/14797585.2011.594582.

(2014), 'Metal Beyond Metal'. Souciant. http://souciant.com/2014/01/metal-beyond-

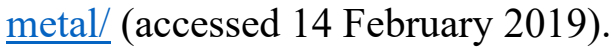

Lilja, E. (2004), 'Characteristics of heavy metal Chord structures', Licentiate thesis, Helsinki: University of Helsinki.

(2009), Theory and Analysis of Classic Heavy Metal Harmony, Helsinki: IAML Finland.

Lucas, O. (2018), “'So Complete in Beautiful Deformity": Unexpected Beginnings and Rotated Riffs in Meshuggah's obZen', Music Theory Online, 24: 3, http://mtosmt.org/issues/mto.18.24.3/mto.18.24.3.lucas.html.

Moore, A. (2001), Rock, the Primary Text: Developing a Musicology of Rock. Brookfield, Vt.: Ashgate.

Mulder, D. (2014), 'Automatic Classification of Heavy Metal Music', Bachelor Thesis, Amsterdam: Universiteit van Amsterdam. 
Mynett, M. (2016), 'The Distortion Paradox: Analyzing Contemporary Metal Production', Studies' in K. Spracklen, A. Brown, K. Kahn-Harris, N.W.R. Scott (eds.), Global Metal Music and Culture: Current Directions in Metal Studies, New York:

Routledge, pp. 68-86.

O'Boyle, T. and Scott, N. (2016), 'The Future of Metal Is Bright and Hell Bent for Genre Destruction: A Response to Keith Kahn-Harris', in K. Spracklen, A. Brown, K. KahnHarris, N.W.R. Scott (eds.), Global Metal Music and Culture: Current Directions in Metal Studies, New York: Routledge, pp. 333-342.

Phillipov, M. (2012), Death Metal and Music Criticism, Lanham, MD: Lexington Books.

Pillsbury, G. (2006), Damage Incorporated: Metallica and the Production of Musical Identity, New York: Routledge.

Purcell, N. (2003), Death Metal Music: The Passion and Politics of a Subculture, Jefferson, N.C: McFarland.

Savigny, H. and Schaap, J. (2018), 'Putting the 'studies' back into metal studies', Metal Music Studies, 4: 3, pp. 549-557, https://doi.org/10.1386/mms.4.3.549_1.

Scotto, C. (2016), 'The Structural Role of Distortion in Hard Rock and Heavy Metal', Music Theory Spectrum, 38: 2, pp. 178-199.

Schumann, G. (2015), 'Terminally Climactic Forms in Heavy Metal Music', in T-M. Karjalainen and K. Kärki (eds) Modern Heavy Metal: Markets Practices and Cultures, Helsinki, Finland: International Institute for Popular Culture.

Smialek, E. (2015), 'Genre and expression in extreme metal music, ca. 1990-2015', Ph.D. dissertation, Montreal: McGill University.

Spracklen, K. (2015), ""To Holmgard... and Beyond": Folk Metal Fantasies and Hegemonic White Masculinities', Metal Music Studies, 1: 3, pp. 354 - 377, https://doi.org/10.1386/mms.1.3.359_1.

Tsatsishvili, V. (2011), 'Automatic Subgenre Classification of Heavy Metal Music', Master's Thesis, Jyväskylä: University Of Jyväskylä.

Walser, R. (1993), Running with the Devil: Power, Gender and Madness in Heavy Metal Music, Hanover, NH: University Press of New England.

Weinstein, D. (2000), Heavy Metal: The Music and Its Culture, 2nd ed., New York: Da Capo Press.

(2016), 'Reflections on Metal Studies' in K. Spracklen, A. Brown, K. Kahn-Harris, N.W.R. Scott (eds.), Global Metal Music and Culture: Current Directions in Metal Studies, New York: Routledge, pp. 22-31.

\section{Acknowledgements}

I am thankful to reddit user $\mathrm{u} / \mathrm{brutishbloodgod} \mathrm{who} \mathrm{first} \mathrm{introduced} \mathrm{me} \mathrm{to} \mathrm{the} \mathrm{idea} \mathrm{of}$ polysemous genre labels and their relevance to metal subgenres. I am thankful for their conversations that helped shape my thinking in this area and for the analogy that illustrates how genre labels can be used that I have adapted with their permission. I am also thankful to 
the anonymous reviewers for their helpful and constructive feedback on the initial version of this paper and also to Jessica Hillier, Carolyn Philpott, and Owen Pearson for their time and effort proofreading the paper and offering advice on early drafts of the model.

\footnotetext{
${ }^{1}$ These bands do share characteristics, otherwise genres labels would be nonsensical. However, their characteristics are not always obvious, concrete or unique making it difficult to group or categorise bands solely based on their characteristics. For example, while harsh vocals are a usually considered a characteristic of death metal, they can also be found in almost any extreme metal subgenre (though their manifestation may differ in other sub-genres). The same can be true for characteristics such as heavily distorted guitars, down-tuning, dissonant riffs etc. Consequently, 'correct' genre labels tend to rise from the canon of bands that are included, meaning that one can accrue subcultural capital by correctly labelling a given band as 'death metal' because the ability to correctly label the band indicates one's knowledge and familiarity with the metal canon. Given the role that genre labels play in accruing authenticity within metal communities, this is perhaps part of the reason why discrete genre characteristics have been slow to develop. See Smialek, 2015 for further elaboration on this idea. ${ }^{2}$ Satanic themes are still common in black metal, yet they are no longer exclusive or compulsory (despite a subset of black metal fans and musicians that insist that Satanism is non-negotiable for 'true' black metal).

${ }^{3}$ While there are differences in musical attributes between metal and these related genres, many of these are also rejected by metal fans for issues of cultural or thematic unsuitability. See Smialek, (2015: 65-115) for a discussion of what he terms 'abject genres' and the potential reasons for their exclusion within metal fan communities.

${ }^{4}$ Within my framework grindcore would properly be considered a hybrid subgenre, as it is a fusion of various subgenres such as death metal, thrash metal, and hardcore punk. However, I have also included it as a major subgenre because of its prominence within the literature as a key subgenre of extreme metal (Kahn-Harris, 2007; Phillipov, 2012; Smialek, 2015).

${ }^{5}$ This refers to the earlier style of doom metal developed by Black Sabbath and exemplified by bands like Witchfinder General, Pentagram, Saint Vitus and Candlemass. While most other subgenres of doom are more closely identifiable with extreme metal, traditional doom is much more closely associated with heavy metal attributes, hence its inclusion under Heavy Metal.

${ }^{6}$ I use this label to refer to the subgenre of black metal pioneered by Bathory's albums Blood Fire Death (1988) and Hammerheart (1990) and developed by bands such as Enslaved. It entails a combination of slower-paced black metal, Nordic/Scandinavian folk music, and Viking-themed paratexts. This is distinct from its other common usage in metal where it refers to bands of any metal subgenre who use Viking-themed paratexts as a core element of their visual or lyrical aesthetic regardless of their musical style (e.g. Amon Amarth, Tyr). ${ }^{7}$ The examples provided in the diagrams are intended to clarify the genre labels for readers who may not otherwise be familiar with certain subgenres. They are not intended to be exhaustive examples. Where there are bands that may fit within multiple categories, I have included them under the heading that is most relevant to the diagram. Some bands are filed under slightly different subgenres in Figure 1 than they are in Figures 2 and 3. This is because they provide a broad example of the subgenre in Figure 1, demonstrating the breadth of the major genre labels, while they give an example of more specific subgenres in Figures 2 and 3.

${ }^{8}$ This idea is analogous to Berger's observations about temporality and perception in metal drumming (Berger, 1997). He notes that different temporal elements in the performance of metal drumming are foregrounded depending on the listener's perception although all elements are always present in the performance itself. This explanation was very helpful in considering how the model proposed in the article relates to active musical objects.

9 'Meaning' is a vague term within most scholarship where it can infer anything from the structural or harmonic function of a set of pitches to the representation of social, cultural or narrative elements within a given musical work. In this case, the model facilitates analysis that demonstrates how a series of musical and extra-musical characteristics 'mean' a certain genre within a given context. As the definitions of 'meaning' within scholarship on metal can be diverse, it is hoped that the model can address this diversity and connect the disparate 'meanings' together through the features of metal genres, because a coherent statement of what something 'means' in metal music should ideally account for all of the various elements that comprise it.

${ }^{10}$ Elflein discusses these issues of rhythmic organisation in metal at length $(2010 ; 2013)$. One of the factors complicating this is the 'poverty of Western thinking about rhythm' when applied to rock, and, by extension, music (Gracyk, 1996: 147). While this is an area still developing within metal musicology, rhythm remains an important element of musical style and genre in metal. Furthermore, the decision to represent a riff organised within meter (or not) is an interpretive decision to be made by the transcriber; similar issues regarding the
} 
transcription of music are encountered by any transcriber who uses standard Western musical notation to represent any genre of music that is not within the Western Art tradition. Many of these issues are concisely addressed by Lilja (2009: 18-20).

${ }^{11}$ As this model has not yet been widely used, the examples will be drawn from the existing literature because establishing conclusively the genre characteristics of more obscure subgenres (e.g. war metal) would require extensive examples beyond the scope of this article.

${ }^{12}$ As Smialek (2015: 195) notes, Verse-Chorus forms are not inherently less complex than other structures but are both ubiquitous and popular with songwriters and listeners in broader Popular music genres. Tellingly, those extreme metal subgenres that employ Verse-Chorus forms alongside other stylistic elements (such as melodic death metal) experience higher mainstream popularity, perhaps influenced by the usage of this structure (Ibid: 234-5). 